\section{P. Kamtsiuris}

K. E. Bergmann

A. Dippelhofer

H. Hölling

B.-M. Kurth

W. Thefeld
Der Pretest des Kinder- und Jugendgesundheitssurveys: Methodische Aspekte und Durchführung

The Pilot Study of the National Health Interview and Examination Survey for Children and Adolescents: Methodical Aspects and Procedure

\section{Zusammenfassung}

In den vom Robert Koch-Institut eigenverantwortlich durchgeführten Pretest des Kinder- und Jugendgesundheitssurveys wurden vom 12. März 2001 bis zum 15. März 2002 insgesamt 1630 Kinder und Jugendliche im Alter zwischen 0 und 18 Jahren sowie deren Eltern einbezogen. Hierbei spielten insbesondere methodische Anliegen eine wichtige Rolle. So wurden Befragungsinstrumente hinsichtlich ihrer Eigenschaften getestet, Indikatoren (beispielsweise für die psychische Gesundheit) entwickelt und evaluiert, verschiedene „Feldzugänge“ (d. h. Zugänge zu den Studienteilnehmern) sowie Methoden zur Erhöhung der Motivation für eine Teilnahme an der Studie erprobt und die Verallgemeinerungseigenschaften bzw. Validität der erhaltenen Informationen untersucht. Bei der Durchführung des Pretests wurden zahlreiche Erfahrungen und Erkenntnisse gewonnen, deren Umsetzung insgesamt zu einer Optimierung der Vorgehensweise in der Hauptphase des Surveys beitragen wird.

\section{Schlüsselwörter}

Gesundheitssurvey · Kinder und Jugendliche · Pretest

\section{Abstract}

In the pilot study of the National Health Survey for Children and Adolescents which has been conducted under the responsibility of the Robert Koch Institute from March $12^{\text {th }} 2001$ to March $15^{\text {th }}$ $2002,1,630$ children and adolescents from 0 to 17 years as well as their parents have been included. Methodological issues have been very prominent. Questionnaires regarding properties, indicators (for example for psychic well-being) were developed and evaluated, different methods of access to the study participants as well as methods to increase the motivation to participate in the study were tested, and the property for generalisation or validity of the obtained information was investigated. The conduct of the pilot study resulted in a number of experiences and findings that will contribute to an optimised approach in the main course of the survey.

Key words

Health survey · children and adolescents · pilot study
Im Juni 2000 wurde einem vom Bundesministerium für Bildung und Forschung (BMBF) und Bundesministerium für Gesundheit (BMG) bestellten Gutachtergremium ein Projektantrag für einen Kinder- und Jugendgesundheitssurvey vorgelegt. Auf der Grundlage eines positiven Gutachtens sowie der Zustimmung der Beauftragten für den Datenschutz und des positiven Votums der Ethik-Kommission der Humboldt-Universität bekam das Robert Koch-Institut den Auftrag, einen zwölf Monate währenden Pre- test durchzuführen. Dieser Pretest wurde vom BMBF finanziert und von einem Wissenschaftlichen Beirat begleitet. Ziel der Pilotstudie war es, im Ergebnis einen auf Machbarkeit, Akzeptanz und Qualität geprüften Projektantrag für den Hauptsurvey, vorgesehen mit ca. 18000 Kindern und Jugendlichen im Alter zwischen 0 und 18 Jahren, vorzulegen.

Zu allen Fragestellungen hat der Pretest zielführende Informationen geliefert, die eine Entscheidung zur Vorgehensweise in einem Hauptsurvey ermöglichen. Ausführungen und Ergebnisse

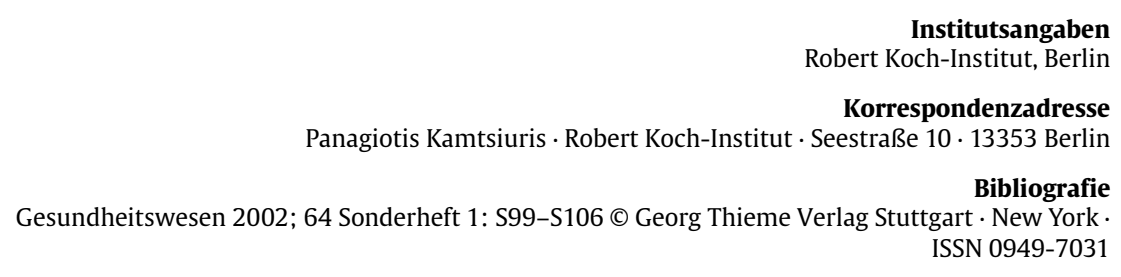




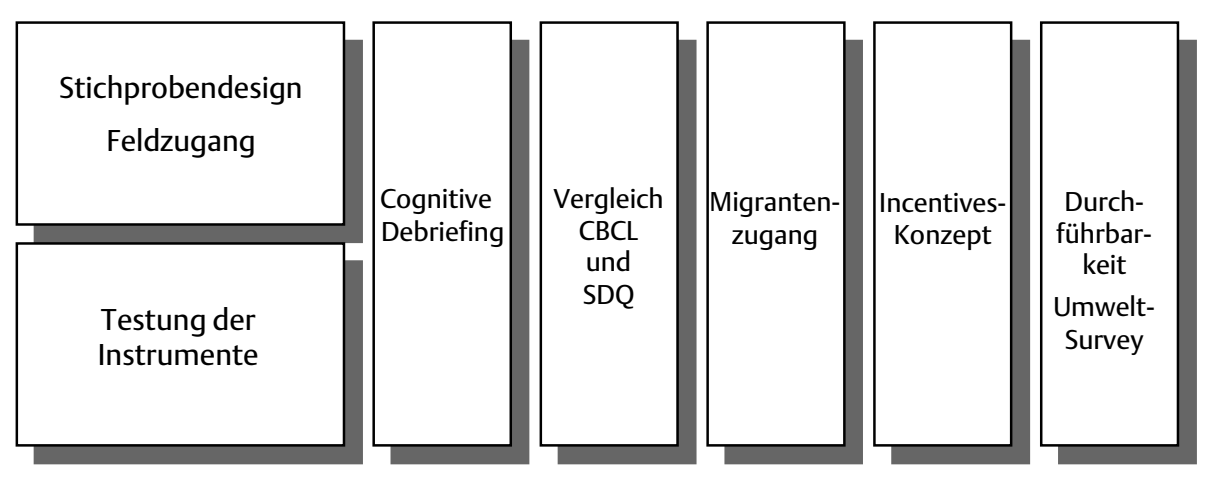

Abb. 1 Methodische Aspekte des Pretests.

zu einzelnen ausgesuchten Themen finden sich in einzelnen Beiträgen dieses Sonderheftes. In dem hier vorliegenden Beitrag wird der Pretest des Kinder- und Jugendgesundheitssurveys in seinen wesentlichen Parametern hinsichtlich Methodik und Durchführung beschrieben.

\section{Methodische Aspekte}

Die wesentlichen methodischen Gesichtspunkte des Pretests für einen Kinder- und Jugendgesundheitssurvey sind in Abb. 1 zusammengefasst.

\section{Stichprobendesign und Zusammensetzung der Teilnehmergruppe}

Die Grundgesamtheit (Zielpopulation) des Pretests umfasste alle Kinder und Jugendlichen im Alter zwischen 0 und 18 Jahren, die in den vier ausgesuchten Standorten Berlin-Steglitz, BerlinFriedrichshain, Neuruppin (Brandenburg) und Wesendorf (Niedersachsen) lebten. Ausgeschlossen waren dabei Kinder und Jugendliche in Einrichtungen, wie z.B. Krankenhäuser, Heil- und Pflegeanstalten.

Im Zuge der Stichprobenplanung für den Kinder- und Jugendgesundheitssurvey wurden im Pretest verschiedene Varianten der Stichprobenrekrutierung hinsichtlich ihrer Realisierbarkeit überprüft. Zur Auswahl standen die Ziehung einer Einwohnermelderegisterstichprobe und die einer Klumpenstichprobe von Schulen bzw. Schulklassen für die Altersgruppe 11 bis 15 Jahre.

Die Einwohnermelderegisterstichprobe folgte den Prinzipien eines geschichteten Random-Designs: In jedem der vier Sample Points wurden die Stichprobeneinheiten zuerst nach dem Alter stratifiziert. Über die Einwohnermeldeämter wurden dann aus jeder Schicht (Strata), d. h. für die einzelnen Jahrgänge 0 bis 17, gleiche Anzahlen von Personenadressen zufällig gezogen. Die Ziehung aus den Adressdateien der Einwohnermeldeämter erfolgte hierbei nach einem mathematischen Zufallsverfahren (systematische Zufallsauswahl mit Startzahl und vorgegebenem Intervall).

Geplant war eine Bruttostichprobe von 2816 Probanden. Im Laufe des Pretests zeigte sich jedoch, dass die geplante Anzahl von 50 Untersuchungen pro Woche nicht einzuhalten war, so dass schließlich, in den 32 vorgesehenen Untersuchungswochen für diese Variante, 2479 Kinder und Jugendliche zu einer Teilnahme am Survey eingeladen wurden. Die Verteilung der geplanten und der tatsächlich realisierten Gesamtnettostichprobe auf die einzelnen Altersstufen wird durch Tab. 1 wiedergegeben. Die Verteilung der Nettostichprobe über die einzelnen Altersgruppen entspricht in etwa der geplanten Struktur.

Tab. 1 Geplante und tatsächlich realisierte Nettostichprobe - Variante Einwohnermelderegister

\begin{tabular}{|c|c|c|}
\hline Altersgruppe (in Jahren) & $\begin{array}{l}\text { geplante } \\
\text { Nettostichprobe }\end{array}$ & $\begin{array}{l}\text { realisierte } \\
\text { Nettostichprobe }\end{array}$ \\
\hline & Anzahl (Anteil) & Anzahl (Anteil) \\
\hline $0-2$ & $240(15,4 \%)$ & $171(13,8 \%)$ \\
\hline $3-6$ & $360(23,1 \%)$ & $265(21,5 \%)$ \\
\hline $7-10$ & $320(20,5 \%)$ & $290(23,5 \%)$ \\
\hline $11-13$ & $288(18,5 \%)$ & $247(20,0 \%)$ \\
\hline $14-17$ & 352 ( $22,6 \%)$ & $262(21,2 \%)$ \\
\hline gesamt 0-17 & 1560 (100\%) & 1235 (100\%) \\
\hline
\end{tabular}

Von den 2479 eingeladenen Probanden waren 290 (11,7\%) als qualitätsneutrale Ausfälle einzustufen. Schließlich konnten 1235 rekrutierte Probanden über die Einwohnermelderegister untersucht werden. Die Gesamtresponse der Ziehungsvariante Einwohnermelderegister beträgt $57 \%$ und liegt entsprechend unterhalb der angestrebten 60\%. Die Nicht-Erreichung der Zielgröße von 60\% Nettoresponse hängt auch von der Erprobung eines Konzeptes zur Gewinnung von Probanden und der damit verbundenen Einführung von Kontrollwochen ab.

Die Schulenstichprobe für den Altersbereich 11 bis 15 Jahre folgte den Prinzipien eines geschichteten Random-Cluster-Designs. Das Stichprobendesign ist bei dieser Variante eine Kombination aus einem geschichteten Random-Design und einem Cluster-Design mit folgenden Auswahlstufen: Die Schulen wurden in den einzelnen Sample Points nach Schultyp geschichtet. Aus jeder Schicht wurde eine Schule zufällig ausgesucht. In jeder der gezogenen Schulen wurde dann aus einer bestimmten Klassenstufe eine Klasse (Cluster) zufällig gezogen. Alle Schüler der ausgewählten Klassen bildeten die Bruttostichprobe. Die Tab. 2 gibt für die Schulvariante die realisierte Bruttostichprobe sowie die geplante und tatsächlich realisierte Nettostichprobe an.

Insgesamt wurden in der Schule 395 Jugendliche im Alter von 11 bis 17 Jahren untersucht und befragt. Nur 355 dieser Probanden lagen in dem vorgesehenen Altersbereich von 11 bis 15 Jahren. 
Tab. 2 Geplante und tatsächlich realisierte Nettostichprobe - Variante Schule

\begin{tabular}{|c|c|c|c|}
\hline Alter (in Jahren) & $\begin{array}{l}\text { geplante } \\
\text { Nettostichprobe }\end{array}$ & $\begin{array}{l}\text { realisierte } \\
\text { Bruttostichprobe }\end{array}$ & $\begin{array}{l}\text { realisierte } \\
\text { Nettostichprobe }\end{array}$ \\
\hline 11 & $96(20 \%)$ & $84(15,5 \%)$ & $59(14,9 \%)$ \\
\hline 12 & $96(20 \%)$ & $101(18,6 \%)$ & $72(18,2 \%)$ \\
\hline 13 & 96 ( $20 \%)$ & 108 ( $19,9 \%)$ & 86 ( $21,8 \%)$ \\
\hline 14 & 96 ( $20 \%)$ & 83 ( $15,3 \%)$ & $58(14,7 \%)$ \\
\hline 15 & 96 ( $20 \%)$ & $116(21,4 \%)$ & $80(20,3 \%)$ \\
\hline$>15$ & $0(0 \%)$ & $51(9,4 \%)$ & $40(10,1 \%)$ \\
\hline gesamt: $11-17$ & & $543(100 \%)$ & $395(100 \%)$ \\
\hline $11-15$ & 480 (100\%) & & 355 \\
\hline
\end{tabular}

Die Gesamtresponse der Zugangsvariante Schule beträgt bei den 11 - bis 15 -Jährigen $73 \%$.

Die Bewertung der beiden Stichprobendesigns erfolgte auf der Basis folgender Kriterien: logistische Unterschiede und Kosteneffizienz der Feldzugänge, Informationsbedarf und Komplexität bei der Stichprobenziehung, Ausschöpfungsquoten der Varianten, Stichproben-Bias und Repräsentativität der Stichproben sowie Stichprobenfehler und Präzision der Ergebnisse. Aufgrund dieser Bewertung wurde für den gesamten Altersbereich 0 bis 17 Jahre die Einwohnermelderegisterstichprobe gewählt [Kamtsiuris, Lange 2002].

\section{Testung der Erhebungsinstrumente}

Bei der Erprobung der Erhebungsinstrumente in der Pilotphase war ein wesentlicher Gesichtspunkt, deren Eignung im Rahmen der räumlichen und zeitlichen Vorgaben sowie der Altersstruktur der Probanden zu überprüfen. Darüber hinaus wurden die Standardisierbarkeit der Methode und die Akzeptanz durch die Teilnehmer und deren Eltern bewertet. Die Daten wurden vor allem im Hinblick auf die Validität und Plausibiltät der Ergebnisse ausgewertet. Tab. 3 gibt einen Überblick über die Art und Themen der getesteten Erhebungsinstrumente im Pretest wieder.
Ein integrierter Bestandteil des Gesamtuntersuchungsablaufs im Pretest des Kinder- und Jugendgesundheitssurveys war die interviewerassistierte schriftliche Befragung. Den einzelnen Altersbereichen angepasst (0-2 Jahre, 3-6 Jahre, 7-10 Jahre, 11-13 Jahre und 14-17 Jahre) kamen insgesamt sieben Selbstausfüllfragebogen zum Einsatz. Die Befragung erfolgte zum einen über die Eltern bzw. Erziehungsberechtigten und zum anderen wurden parallel dazu auch die Kinder ab dem 12. Lebensjahr mit zwei unterschiedlichen, altersangepassten Fragebogen (11-13 Jahre, 14-17 Jahre) um Auskunft gebeten. Die Inhalte der Fragebogen orientierten sich an wesentlichen gesundheitsrelevanten Fragestellungen, wie akute und chronische Krankheiten, Schmerzen, Unfälle, psychische Gesundheit und Lebensqualität, Freizeitverhalten, Inanspruchnahme medizinischer Leistungen und Ernährung. Die Auswertung der erhobenen Daten ergab insgesamt eine gute Akzeptanz der Fragebogen. Hinsichtlich Plausibilität, Validität und Vollständigkeit wiesen sich die Fragebogen als geeignetes Surveyinstrument aus und werden nach geringen Adaptationen auch im Hauptsurvey zum Einsatz kommen [Knopf et al. 2002, Neuhauser et al. 2002, Thefeld et al. 2002].

Bei den körperlichen Untersuchungen erwiesen sich die Feldbedingungen (Umgebungslärm, Unruhe durch das Kind oder die Begleitpersonen) und die Altersstruktur der Probanden als bestimmend für die Auswahl der Messmethode für den Blutdruck. Da die Quecksilbermanometermethode, wie sie im ersten Teil des Pretests durchgeführt wurde, nur bei exakter Auskultation zu validen Ergebnissen führte, wurde im zweiten Teil die Oszillometrie eingesetzt. Mit dieser Methode konnten standardisiert über das gesamte kindliche Altersspektrum hinweg zuverlässige Blutdruckwerte ermittelt werden. Bei der Untersuchung der Haut konnte das Ziel, alle Hauterkrankungen standardisiert erfassen zu können, nicht erreicht werden. Die Diagnose vieler Hauterkrankungen ist nur Spezialisten vorbehalten und erwies sich zudem als sehr zeitaufwändig. Die Beurteilung von Schweregrad und Ausdehnung der Neurodermitis mit einem bewährten Instrument (SCORAD) konnte jedoch problemlos umgesetzt werden. Als limitierend bei der Reifebestimmung erwies sich die Akzeptanz der Methode durch die Teilnehmer. Die Fremd-

Tab. 3 Erhebungsinstrumente im Pretest

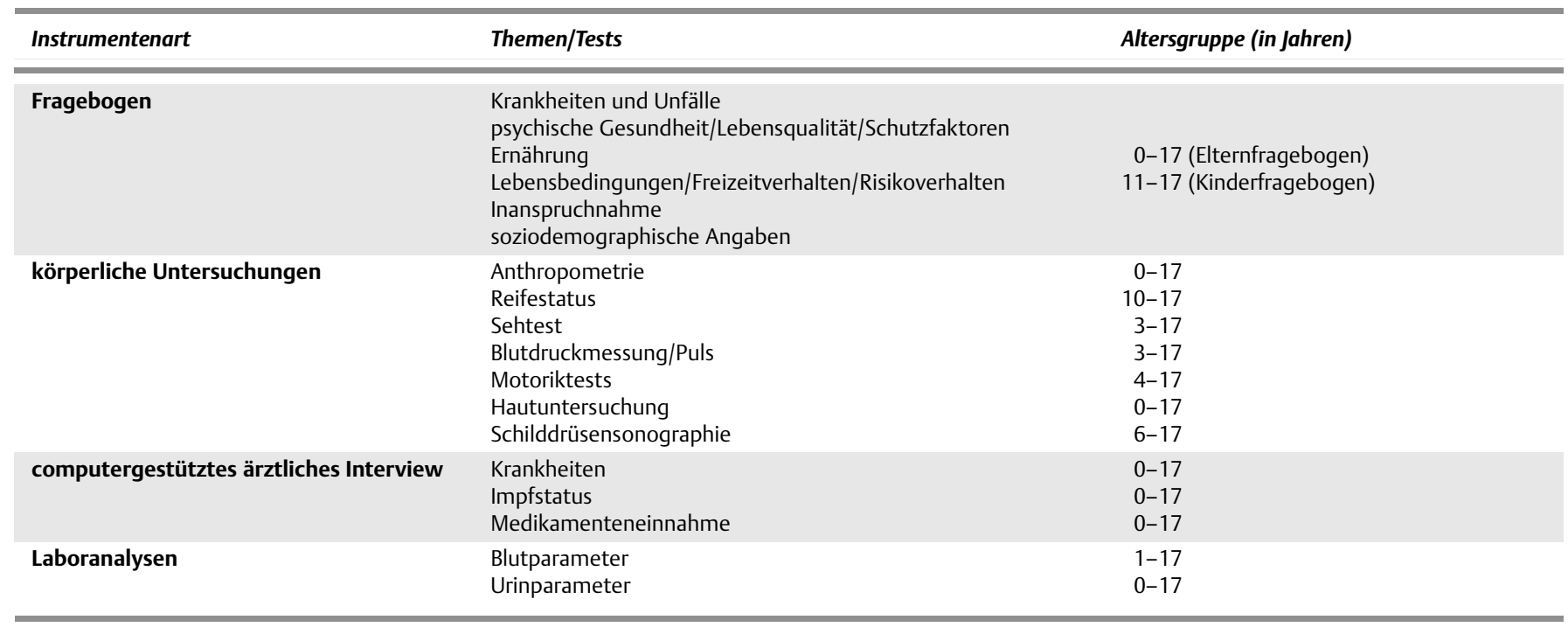


beurteilung der Schambehaarung durch die Ärztin/den Arzt wurde von einem großen Teil der Probanden abgelehnt, die Selbsteinschätzung mittels Tafeln wurde hingegen gut angenommen. Auch die anderen Untersuchungsteile (Sehtest, motorische Tests, Anthropometrie und Schilddrüsensonographie) wurden kritisch getestet und in der Durchführung optimiert bzw., wenn erforderlich, auch inhaltlich überarbeitet [Dippelhofer et al. 2002a, Dippelhofer et al. 2002b, Kahl, Emmel 2002].

Ein weiteres Ziel des Pretests war es, die ärztliche Befragung zu Krankheiten mittels computerassistiertem persönlichem Interview (CAPI) als Instrument zu prüfen und zu verbessern. Darüber hinaus wurden das Spektrum und die Verteilung der Fragen zu akuten und chronischen Erkrankungen auf den Selbstausfüllfragebogen oder im ärztlichen Interview optimiert.

Aufgrund der besonderen rechtlichen Position der Studienteilnehmer als nicht oder nur bedingt einwilligungsfähige Personen ist die Blutentnahme im Vorfeld des Pretests kritisch diskutiert und von der Ethikkommission an bestimmte Auflagen gebunden worden. Es musste ein schriftliches Einverständnis der Eltern und ab 14 Jahren auch das der Jugendlichen vorliegen. Des Weiteren sollte nicht gegen den erklärten Willen des Kindes oder Jugendlichen Blut abgenommen oder bei einer Fehlpunktion ein Wiederholungsversuch durchgeführt werden. Zur Betäubung der Haut wurde allen Probanden ein anästhesierendes Pflaster angeboten. Es hat sich in der Praxis herausgestellt, dass diese Vorgaben problemlos eingehalten werden konnten und eine außerordentlich hohe Responserate von über $90 \%$ erreicht werden konnte. Nicht selten waren die erwarteten Ergebnisse der umfangreichen Blutuntersuchung das maßgebliche Kriterium für die Teilnahme an der Studie.

\section{Cognitive Debriefing}

Die Fragebogen wurden vor Beginn des eigentlichen Pretests einem Cognitive Debriefing unterzogen. Hierbei wurde in enger Zusammenarbeit mit Mitarbeitern des ZUMA eine methodische Erhebung in Form eines Zwei-Phasen-Pre-Pretests entwickelt und durchgeführt [Prüfer, Rexroth 1996]. Die Verständlichkeit und Akzeptanz der Fragebogen wurden getestet, die Zeitdauer des Ausfüllens wurde gemessen und es wurde geprüft, ob 10-jährige Kinder den Selbstausfüllfragebogen beantworten können.

In der ersten Phase wurde ein kognitives Interview bei 25 Elternteilen und 20 Kindern bzw. Jugendlichen durchgeführt. Aktive kognitive Techniken, wie z.B. „Probing“ (Hinterfragen von Antworten) oder „Think aloud“ (die befragte Person wird aufgefordert, während oder nach der Beantwortung der Frage Überlegungen laut $\mathrm{zu}$ formulieren), wurden dabei angewandt. In der zweiten Phase, dem Standard-Pre-Pretest, wurden die Durchführbarkeit und Akzeptanz des ganzen Instruments bei 40 Teilnehmern (25 Erwachsenen und 15 Kindern bzw. Jugendlichen) erprobt. In dieser Phase hinterfragte der Interviewer nicht aktiv, sondern beobachtete die Probanden und protokollierte Zeitdauer, Reaktionen, Probleme und Auffälligkeiten beim Ausfüllen. Abschließend wurden die Probanden um eine Beurteilung und Benotung des Fragebogens gebeten.
Die Probanden überschritten mit durchschnittlich 76 Minuten (35-120 Minuten, Median 75 Minuten) die zum Ausfüllen der Fragebogen vorgesehene Zeit, so dass diese um 15 bis 20\% gekürzt wurden. Die 10-jährigen Kinder waren nicht in der Lage, einen Selbstausfüllbogen zu beantworten. Im Weiteren zeigte sich, dass den Probanden sowohl Begriffe aus einzelnen Fragen als auch ganze Fragen unverständlich waren; daher wurden Formulierungen geändert und Fragen herausgenommen. Insgesamt war die Akzeptanz der Fragebogen jedoch gut, im Durchschnitt wurden sie mit der Schulnote 2,5 beurteilt.

\section{Methodischer Vergleich der Instrumente CBCL und SDQ}

Das Ziel, psychisch auffälliges Verhalten von Kindern und Jugendlichen mit international entwickelten Instrumenten in kürzerer Form zu erfassen, kann entweder durch den Einsatz der Child Behavior Checklist (CBCL) [Arbeitsgruppe Deutsche Child Behavior Checklist 1998a, Arbeitsgruppe Deutsche Child Behavior Checklist 1998b] oder den Strengths and Difficulties Questionnaire (SDQ) [Goodmann 1997] realisiert werden. Die Eignung beider Instrumente für epidemiologische Zwecke wurde im Pretest in einer Zusatzbefragung zu psychischer Gesundheit und Wohlbefinden hinsichtlich Reliabilität, Validität und Spezifität miteinander verglichen [Bettge et al. 2002]. Hierzu wurde während der Datenerhebung des Pretests eine Untergruppe mit einem Umfang von 414 Kindern und Jugendlichen im Alter von 6 bis 17 Jahren bzw. deren Eltern für den methodischen Vergleich beider Instrumente in die Zusatzbefragung einbezogen. Zur Beurteilung der klinischen Aussagekraft beider Instrumente wurde zusätzlich im einem Fall-Kontroll-Design mit 155 Kindern und Jugendlichen sowie deren Eltern ein psychodiagnostisches Interview durchgeführt. Die Datenauswertung erfolgte im Hinblick auf die psychometrische Qualität (Reliabilität, Validität), den diagnostischen Wert und die Praktikabilität der eingesetzten Instrumente. Anhand der Ergebnisse wurde entschieden, die SDQ als Instrument zur Erfassung von psychischen Auffälligkeiten in der Hauptphase des Surveys einzusetzen.

\section{Zugang zu Migranten}

Analysen des Teilnahmeverhaltens von Migranten beziehen sich bisher ausschließlich auf Untersuchungen, die mit der Erhebungsmethode „mündliches Interview“ realisiert wurden [Koch 1997]. Zur Teilnahmebereitschaft von Migranten an Gesundheitsstudien mit medizinischem Untersuchungsteil liegen keine systematischen Studien vor. Es lassen sich aber zusätzliche $\mathrm{Zu}$ gangsbarrieren vermuten, da der Anteil der Probanden ausländischer Herkunft in den Netto-Stichproben noch geringer ausfällt als bei „reinen“ Befragungen. So macht der Anteil der Probanden mit nichtdeutscher Staatsangehörigkeit im BundesGesundheitssurvey (BGS) von 1998 lediglich 4,8\% aus; das entspricht nur etwa der Hälfte ihres damaligen Anteils in der Bevölkerung $(8,9 \%)$.

Untersuchungsleitend war für den Pretest des Kinder- und Jugendgesundheitssurveys die Fragestellung, wie Zugangswege, Erhebungsinstrumente und die Durchführung der Feldarbeit für Teilnehmer nichtdeutscher Herkunftssprache optimiert werden können. Im Ergebnis wurden Bedingungen formuliert, die erfüllt sein müssen, damit Migranten an der Studie teilnehmen können (trotz Sprachbarrieren) und einer ärztlichen Untersuchung bzw. 
einem Hausbesuch auch zustimmen (trotz kultureller und anderer Vorbehalte) [Schenk 2002].

\section{Wirkung von Incentives auf die Teilnahmebereitschaft}

Während der Pretestphase wurde die Wirkung von Incentives auf die Teilnahmebereitschaft getestet. Hierbei sind unterschiedliche Arten von Geschenken (nichtmonetär versus monetär, Höhe des Geldbetrages) sowie die Methode der Übergabe (mitgeschickt versus „versprochen“) getestet worden. Die methodische Erprobung verlief in zwei Phasen.

In der ersten Phase, der Informationsgewinnungsphase, wurden die Präferenzen der Probanden hinsichtlich einer Aufwandsentschädigung für eine Teilnahme an dem Survey ermittelt. Dafür wurden standardisierte telefonische Interviews bei einer jeweils gleichen Anzahl von Teilnehmern und Nicht-Teilnehmern durchgeführt, die zufällig aus der Brutto-Stichprobe gezogen wurden. Durchgeführt wurden 183 telefonische Interviews im Zeitraum von August bis Oktober 2001. Um alters- und regionsspezifische Präferenzen zu berücksichtigen, wurden die Befragungen über fünf Altersgruppen und die vier Standorte verteilt.

In der zweiten Phase wurden die Ergebnisse der Informationsgewinnungsphase umgesetzt und evaluiert. Hierbei erstreckte sich die Erprobungsphase über die gesamte Dauer der zweiten Hälfte des Pretests (Oktober 2001 bis März 2002). Das Design zur Prüfung der Wirkung von Incentives beinhaltete drei Versuchsgruppen und eine Kontrollgruppe. Der Kontrollgruppe wurde im Anschreiben weder ein Sachgeschenk noch eine monetäre Aufwandsentschädigung angekündigt. In der Testgruppe 1 wurde den potenziellen Teilnehmern ein Sachgeschenk mit Angabe der Art und des Wertes versprochen. Ähnlich ist den Bruttoprobanden der Testgruppe 2 eine monetäre Aufwandsentschädigung für den Fall der Teilnahme angekündigt worden. Demgegenüber wurde den Personen in der dritten Gruppe ein Geschenk in Form eines Gutscheins schon bei der Einladung beigelegt. Hierbei war der Wert des Gutscheins halb so hoch wie die angekündigte Belohnung in den anderen beiden Gruppen. [Diekmann und Jann 2001] zeigten in einem Experiment, dass ein beigelegtes Geschenk zu einem Anstieg der Ausschöpfungsquote um ca. 10 Prozentpunkte führt. Die Zuordnung der Probanden in die einzelnen Testgruppen erfolgte per Zufall.

Insgesamt wurden, nach Analyse der erhobenen Daten, Empfehlungen für die Hauptphase des Surveys mit Bezug auf die einzelnen Altersgruppen erarbeitet.

\section{Die Pretestung des Umwelt-Surveys}

Kinder und Jugendliche sind aufgrund ihrer expositionsrelevanten Verhaltensweisen sowie ihrer besonderen physiologischen Bedingungen als besondere Risikogruppe für umweltbedingte Gesundheitsbeeinträchtigungen $\mathrm{zu}$ betrachten. Vom Umweltbundesamt wurde daher ein mit dem Gesundheitssurvey für Kinder und Jugendliche verknüpftes Umwelt-Modul entwickelt und dem Robert Koch-Institut zur Pretestung in Auftrag gegeben. Mit diesem Projekt sollen die in den Jahren 1985/86, 1990/92 und 1997/99 gemeinsam mit entsprechenden Gesundheitssurveys für die erwachsene Bevölkerung durchgeführten UmweltSurveys fortgeführt und um Daten für Kinder und Jugendliche erweitert werden [Schulz et al. 2002].
Den Schwerpunkt des Untersuchungsprogramms des Pretests bildete dabei das Human-Biomonitoring (Untersuchung körpereigenen Materials), bei dem Blut und Urin des Probanden auf zahlreiche Umweltschadstoffe untersucht wurden. Weiterhin wurden zur Expositionsabschätzung und Charakterisierung der Belastungspfade Untersuchungen im Sinne des Umweltmonitorings im Haushalt der Kinder und Jugendlichen und in der Gemeinde durchgeführt. Hierzu zählten die Untersuchung von Lärm und Hörfähigkeit bei den Probanden, Trinkwasser- und Staubproben im Haushalt, Außenluftmessungen in der Gemeinde sowie die Durchführung standardisierter Interviews mit Eltern und Kindern. In über alle Altersklassen verteilten Unterkollektiven wurden chemische und biologische Luftverunreinigungen untersucht.

Die Untersuchungsinstrumente wurden im Pretest an 550 zufällig ausgewählten Probanden auf Ausschöpfung, Praktikabilität und Compliance untersucht. Die gesamte logistische Infrastruktur des Gesundheitssurveys konnte für das Umwelt-Modul genutzt werden. Dies betraf sowohl die Informations- und Öffentlichkeitsarbeit als auch die Probandenrekrutierung, die NonResponder-Analyse, die Bereitstellung der Räume, die Organisation der Umzüge, den Proben- und Materialtransport sowie die Dateneingabe. Die Ergebnisse des Pretests zur Stichprobenziehung, Repräsentativität, Responserate, zur Zumutbarkeit und Machbarkeit der Feldarbeit, Einsetzbarkeit der Erhebungsinstrumente sowie zur Optimierung des zeitlichen Ablaufs und der Logistik wurden in einem gesonderten Forschungsbericht für das Umweltbundesamt als Auftraggeber ausführlich dargestellt.

\section{Durchführung des Pretests}

Der zeitliche Ablauf des Pretests gliederte sich im Wesentlichen in drei Phasen: die Vorbereitungsphase, die Feldarbeitsphase sowie die Auswertungs- und Präsentationsphase. Es wurde eine Gesamtdauer von 19 Monaten für den Pretest veranschlagt. Die reine Feldarbeitszeit betrug zwölf Monate. In Abb. 2 werden die einzelnen Projektphasen sowie der zeitliche Ablauf dargestellt.

Nach positiver Begutachtung des Projektes durch die Ethikkommission der Berliner Humboldt-Universität (Virchow-Klinikum), den Bundesbeauftragten für den Datenschutz und die zuständigen Datenschutzbeauftragten der Länder Berlin, Brandenburg und Niedersachsen wurden die Organisation und Durchführung der Feldarbeit sowie die Datenerfassung, -aufbereitung und -auswertung des Pretests in eigener Verantwortung des Robert Koch-Instituts realisiert. Der Pretest wurde durch einen wissenschaftlichen Beirat, der aus sieben Mitgliedern bestand, begleitet.

Die externe Qualitätskontrolle wurde nach Ausschreibung durch das Bremer Institut für Präventionsforschung und Sozialmedizin (BIPS) durchgeführt. Zudem fand auch kontinuierlich eine interne Qualitätssicherung durch Mitarbeiter des Robert Koch-Instituts statt.

Die wesentlichen Schwerpunkte der Vorbereitungsphase waren die Rekrutierung der Projektmitarbeiter, die Stichprobenziehung, die Durchführung einer dreiwöchigen Schulung des Feld- 


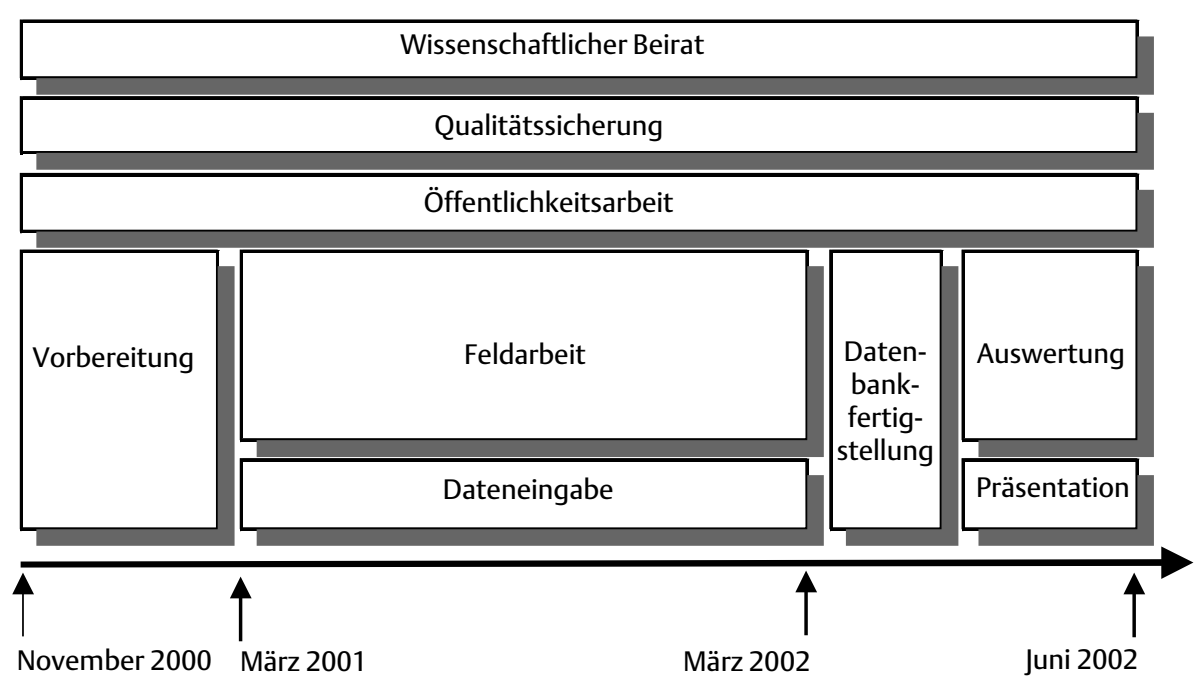

Abb. 2 Phasen und zeitlicher Ablauf des Pretests.

personals sowie die Konzipierung und Vorbereitung der Öffentlichkeitsarbeit.

\section{Routenplan und Personaleinsatz}

Die Feldarbeit des Pretests wurde im Zeitraum von März 2001 bis März 2002 durchgeführt. Einem vor Beginn der Studie festgelegten Routenplan folgend wurde die Untersuchung an vier Standorten (Berlin-Steglitz, Berlin-Friedrichshain, Wesendorf und Neuruppin), jeweils einmal in den Sommer- und einmal in den Wintermonaten, durchgeführt. Die Untersuchungen der Probanden der Einwohnermelderegisterstichprobe wurden vorwiegend in Räumen des Öffentlichen Gesundheitsdienstes (ÖGD), die der Schulstichprobe in den jeweiligen Schulen durchgeführt. Das bedeutete, dass zahlreiche Standorte, davon allein 13 verschiedene Schulen, bezogen und insgesamt 25 Umzüge durchgeführt werden mussten. Die Aufenthaltsdauer in den einzelnen Untersuchungsräumen variierte von drei bis fünf Tagen in den Schulen, bis zu vier Wochen in den Untersuchungszentren des ÖGD.

Das Untersuchungsteam für die Gesundheitsuntersuchung setzte sich aus fünf Mitarbeitern zusammen: der Kinderärztin, dem Zentrumsinterviewer, der Kinderkrankenschwester, der MTA und dem Feldvorbegeher. In das Team integriert waren zwei Umweltinterviewer, die für den Bereich der Umweltuntersuchungen zuständig waren.

Der Transport der Laborproben vom Untersuchungszentrum zum Labor erfolgte dreimal pro Woche und wurde ebenso wie die Nachlieferung von Verbrauchsmaterialien vom Fahrdienst des Robert Koch-Instituts vorgenommen.

\section{Einladungsmodus}

Für die beiden zu testenden Zugänge (Einwohnermeldeamt versus Schule) ergaben sich sowohl für die Probandenrekrutierung als auch für den organisatorischen Ablauf im Vorfeld der Untersuchung unterschiedliche Vorgehensweisen.

Beim Zugang über das Einwohnermeldeamt wurde etwa acht bis zehn Wochen vor dem Untersuchungszeitraum das Gesundheitsamt im jeweiligen Sample Point angeschrieben, über Inhalt und Anliegen des Surveys informiert und um Unterstützung, vor allem durch die Bereitstellung von Räumlichkeiten im Öffentlichen Gesundheitsdienst, gebeten.

In demselben Zeitraum wurde Kontakt zum Einwohnermeldeamt aufgenommen und die jeweilige Stichprobenziehung aus dem Melderegister veranlasst. Nach Übermittlung der Daten und Eingabe in die Datenbank wurden 28 Tage vor dem geplanten Untersuchungsbeginn die Einladungsschreiben an die Familien verschickt. Bei Teilnahmebereitschaft wurden die Eltern gebeten, die beigefügte Antwortkarte portofrei mit der Angabe ihrer Rufnummer zur Vereinbarung eines Untersuchungstermins an das Robert Koch-Institut zurückzusenden. Wurde innerhalb von zehn Tagen keine Antwortkarte erhalten, erfolgte 18 Tage vor dem Untersuchungszeitraum ein Erinnerungsschreiben an die Eltern. Fehlte bis sieben Tage vor dem Untersuchungszeitraum weiterhin eine Rückmeldung, wurde über den Feldvorbegeher versucht, telefonisch Kontakt zum Probanden aufzunehmen. Bei Probanden, deren Rufnummer nicht vorlag bzw. die auch nach mehrmaligen Versuchen telefonisch nicht erreicht werden konnten, wurden zusätzlich Hausbesuche durchgeführt. Nicht-Teilnehmer wurden gebeten, einen Non-Responder-Fragebogen auszufüllen, bzw. wurden bei Ablehnung des Fragebogens als Totalverweigerer registriert.

Der Zugang über die Schule gestaltete sich organisatorisch vergleichsweise schwierig und sehr zeitaufwändig. Durch die jeweils zuständigen Schulbehörden wurde dem Robert Koch-Institut, nach Vorliegen der erforderlichen Genehmigungen durch die Landesdatenschutzbeauftragten sowie die Kultusbehörden, das Schulverzeichnis des Sample Points zur Verfügung gestellt. Mittels eines statistischen Zufallsverfahrens wurden die Schulen nach Schultyp und Klassenstufe gewichtet und ausgewählt. Die gezogenen Schulen wurden angeschrieben und zur Teilnahme an der Studie gebeten. Lehnte eine Schule die Beteiligung ab, wurde die folgende Schule auf der Rangliste kontaktiert. Als Ablehnungsgrund erwies sich bei den angesprochenen Schulen nicht das mangelnde Interesse oder der organisatorische Mehraufwand, sondern hauptsächlich das Fehlen ausreichender Räumlichkeiten. Zeigte sich die Schulleitung interessiert und verfügte die Schule über ausreichende Zimmer, wurde ein detailliertes Informationsschreiben (bestehend aus Anschreiben, Kopie der Zustimmung des Landesdatenschutzbeauftragten und 
der Zustimmung der Senatsverwaltung (Berliner Schulen) bzw. der Kultusministerien (für Neuruppin und Wesendorf)) an die Schulen versandt. Bei endgültiger Zustimmung wurde ein Gespräch mit der Schulleitung und den beteiligten Klassenlehrern vereinbart und die Räume in der Schule wurden besichtigt. In Abstimmung mit der Schulleitung wurden ein bis zwei Termine für Elterninformationsabende vereinbart (je nach Anzahl der ausgewählten Klassen).

\section{Untersuchungsablauf, Dauer und Akzeptanz der Untersuchungen}

Bei der Feldzugangsvariante Einwohnermelderegisterstichprobe wurden im Durchschnitt zehn Probanden in der Zeit von 8.30 18.00 Uhr in das Untersuchungszentrum einbestellt. Nach einer Einweisung durch den Zentrumsinterviewer haben die Eltern und auch die Kinder und Jugendlichen ab 11 Jahren zunächst die Fragebogen ausgefüllt, daran schlossen sich die medizinischen Tests an. Es folgten das ärztliche Interview (CAPI), die Blutabnahme (bei Vorliegen des Einverständnisses der Eltern und des Kindes) und die Schilddrüsensonographie.

Die Untersuchungen in der Schule begannen mit der Befragung der Schüler im Klassenverband. Im Übrigen unterschied sich der Untersuchungsablauf nicht von dem oben geschilderten. Das ärztliche Interview (CAPI) wurde, da die berufstätigen Eltern ihre Kinder häufig nicht zur Untersuchung begleiten konnten, meistens mit den Jugendlichen allein durchgeführt. Konnten die Jugendlichen selbst keine umfassenden Auskünfte geben, erfolgte das Interview telefonisch mit den Eltern.

Nach einer Einarbeitungsphase für das Feldteam wurde vom 18. April 2001 bis zum 18. Juli 2001 an insgesamt 25 Tagen eine Zeitmessung für die unterschiedlichen Untersuchungsteile vorgenommen. Ausgewertet wurden die Daten von 80 zufälligen Probanden im Alter zwischen 0 und 17 Jahren aus der Einwohnermelderegisterstichprobe. Neben der Gesamtaufenthaltsdauer wurde die Zeit erfasst, die die Probanden (die Eltern und die Kinder und Jugendlichen) zum Ausfüllen der Fragebogen benötigten. Die einzelnen Untersuchungen wurden in zwei Blöcken zusammengefasst: Körpermessung (alle von der Kinderkrankenschwester erhobenen Daten: Sehleistung, Blutdruck, Anthropometrie etc.) und CAPI/Blutentnahme etc. (vom Feldarzt zu erhebende Daten).

In Tab. 4 sind die Zeitdauer, die für die einzelnen Untersuchungsblöcke benötigt wurde, und die Gesamtaufenthaltsdauer mit Minimum, Maximum und Mittelwert dargestellt.

Bei allen Untersuchungsblöcken fallen vor allem die Spannweiten auf. Das hängt mit dem in den einzelnen Altersgruppen un- terschiedlichen Programm für die Körpermessungen zusammen, aber auch damit, dass Kinder (abhängig von Alter und individuellen Merkmalen) viel leichter ablenkbar sind und kürzere Konzentrationsspannen haben als Erwachsene. Die Gesamtaufenthaltsdauer war mit im Mittel 122 Minuten im angestrebten Bereich, wobei auch hier die Schwankungsbreiten groß waren.

Während der Feldarbeit wurde bereits der Eindruck gewonnen, dass die meisten Probanden und auch ihre Eltern eine positive Einschätzung von der Untersuchung abgaben. Zur Objektivierung wurde 128 zufällig ausgewählten Probanden aus der Einwohnermelderegisterstichprobe ein Evaluationsfragebogen vorgelegt. Darin wurden die Eltern und ihre Kinder ab elf Jahren befragt zur Verständlichkeit des Anschreibens, zu den Informationen im Vorfeld, den medizinischen Untersuchungen und motorischen Tests, der Blutentnahme sowie zur Verständlichkeit und Länge des Fragebogens. Schließlich wurde auch die Frage gestellt, ob sie bei der Untersuchung wieder mitmachen würden, wenn sie noch einmal die Wahl hätten. Hierbei bejahten über 90\% der Familien die Frage (nur 1,6\% würden auf keinen Fall wieder mitmachen), was für eine hohe Akzeptanz des Surveys spricht.

\section{Umsetzung der Ergebnisse}

Eine so umfassende repräsentative Untersuchung, wie sie der Kinder- und Jugendgesundheitssurvey vorsieht, mit einer Fallzahl von 18000 Personen wurde in Deutschland bisher noch nicht durchgeführt. Die Vorerfahrungen des Robert Koch-Instituts mit den Erwachsenensurveys [Bellach et al. 1998] und mit Erhebungen bei Schülern [Hüttner et al. 1997] waren zwar wesentliche Voraussetzungen für die Konzeption und Planung des Kinder- und Jugendgesundheitssurveys, ein großer Pretest war dennoch erforderlich.

Durch den Pretest wurden Machbarkeit, Studiendesign und Instrumente überprüft, die Logistik wurde optimiert, die Gründe für die Nicht-Teilnahme und die Voraussetzungen für eine hohe Ausschöpfung wurden ermittelt. Die Fallzahlen bzw. die Präzision der Ergebnisse und die Kosten für die Hauptphase ließen sich genauer veranschlagen.

Der Know-how-Transfer vom Pretest in die Hauptphase der Studie wird gewährleistet durch:

- die Erstellung eines Abschlussberichts über den Pretest,

- die Vorlage eines überarbeiteten Operationshandbuches für die Hauptphase,

- die Vorlage eines angepassten Erhebungsprogramms,

Tab. 4 Zeitdauer für die einzelnen Untersuchungsblöcke (in min) und Gesamtaufenthaltsdauer

\begin{tabular}{llrr} 
& Anzahl der Probanden & Minimum & Mittelwert \\
\hline Körpermessungen und Tests im Schwesternzimmmer & 80 & 5 & 27 \\
Blutabnahme/ärztliches Interview etc. & 75 & 5 & 25 \\
Fragebogen: Eltern & 67 & 20 & 65 \\
Fragebogen: Kinder/Jugendliche & 31 & 25 & 110 \\
Gesamtaufenthalt im Untersuchungszentrum & 75 & 55 & 105 \\
\hline
\end{tabular}


- die Durchführung der Schulung der Feldteams und einer internen Qualitätskontrolle in der Hauptphase durch Mitarbeiter des Robert Koch-Instituts,

- die wissenschaftliche Leitung und Verantwortung der Hauptphase sowie die Organisation und Durchführung der Feldarbeit durch das Robert Koch-Institut,

- die Beibehaltung des zum Pretest einberufenen Wissenschaftlichen Beirates auch in der Hauptphase.

Insgesamt wurden bei der Durchführung des Pretests zahlreiche Erfahrungen gewonnen, die zur Optimierung bei der Planung der Feldarbeit für die Hauptphase des Surveys beigetragen haben. Eine wichtige Erkenntnis ist jedoch auch, dass das Konzept für einen bundesweiten Kinder- und Jugendgesundheitssurvey funktioniert hat und die Erhebung von Eltern und Kindern angenommen worden ist. Die Hauptphase des Kinder- und Jugendgesundheitssurveys läuft seit Herbst 2002, die Erhebung der Daten beginnt im Frühjahr 2003 [Kurth et al. 2002].

\section{Literatur}

${ }^{1}$ Arbeitsgruppe Deutsche Child Behavior Checklist. Elternfragebogen über das Verhalten von Kindern und Jugendlichen; deutsche Bearbeitung der Child Behavior Checklist (CBCL/4-18). Einführung und Anleitung zur Handauswertung. 2. Auflage mit deutschen Normen, bearbeitet von Döpfner M, Plück J, Bölte $S$ et al. Köln: Arbeitsgruppe Kinder-, Jugend- und Familiendiagnostik (KJFD) 1998a

2 Arbeitsgruppe Deutsche Child Behavior Checklist. Fragebogen für Jugendliche; deutsche Bearbeitung der Youth Self-Report Form der Child Behavior Checklist (YSR). Einführung und Anleitung zur Handauswertung. 2. Auflage mit deutschen Normen, bearbeitet von Döpfner M, Plück J, Bölte S et al. Köln: Arbeitsgruppe Kinder-, Jugend- und Familiendiagnostik (KJFD) 1998b

${ }^{3}$ Bellach BM, Knopf H, Thefeld W. Der Bundes-Gesundheitssurvey 1997/1998. Gesundheitswesen 1998; 60 (Sonderheft 2): 59-68

${ }^{4}$ Bettge S, Ravens-Sieberer U, Wietzker A et al. Ein Methodenvergleich der Child Behavior Checklist und des Strengths and Difficulties Questionnaire. Gesundheitswesen 2002; 64 (Sonderheft 1): 119-124
${ }^{5}$ Dieckmann A, Jann B. Anreizformen und Ausschöpfungsquoten bei postalischen Befragungen. Eine Prüfung der Reziprozitätshypothese. ZUMA-Nachrichten 2001; 48: 18-27

${ }^{6}$ Dippelhofer A, Bermann KE, Kahl $\mathrm{H}$ et al. Die körperliche Untersuchung im Rahmen des Kinder- und Jugendgesundheitssurveys. Gesundheitswesen 2002a

${ }^{7}$ Dippelhofer A, Thamm M, Thierfelder W. Jodmonitoring im Rahmen des Kinder- und Jugendgesundheitssurveys. Gesundheitswesen 2002b

${ }^{8}$ Goodman R. The Strengths and Difficulties Questionnaire: A research note. Journal of Child Psychology and Psychiatry 1997; 38: 581-586

${ }^{9}$ Hüttner H, Dortschy R, Heß H et al. Körperliche Aktivität unter Berliner Schulkindern. Ergebnisse der Berliner Studie Gesundheit im Kindesalter (GIK II 1994-1995). Bundesgesundheitsblatt 1997; 8: 270-277

${ }^{10} \mathrm{Kahl} \mathrm{H}$, Emmel J. Der Untersuchungsteil Motorik im Pretest des Kinder- und Jugendgesundheitssurveys. Gesundheitswesen 2002; 64 (Sonderheft 1): 114-118

${ }^{11}$ Kamtsiuris P, Lange M. Der Pretest des Kinder- und Jugendgesundheitssurveys: Stichprobendesign. Gesundheitswesen 2002; 64 (Sonderheft 1): 107-113

${ }^{12}$ Knopf H, Bergmann E, Dippelhofer A et al. Der Kinder- und Jugendgesundheitssurvey als Datenquelle zur Beschreibung wesentlicher Aspekte der gesundheitlichen Versorgung im Kindes- und Jugendalter. Gesundheitswesen 2002; 64 (Sonderheft 1): 43-47

${ }^{13}$ Koch A. Teilnahmeverhalten beim Allbus 1994. Soziodemographische Determinanten von Erreichbarkeit, Befragungsfähigkeit und Kooperationsbereitschaft. Kölner Zeitschrift für Soziologie und Sozialpsychologie 1997; 1: 98-122

${ }^{14}$ Kurth BM, Bergmann KE, Hölling H et al. Der bundesweite Kinder- und Jugendgesundheitssurvey - Das Gesamtkonzept. Gesundheitswesen 2002; 64 (Sonderheft 1): 3-11

${ }^{15}$ Neuhauser H, Dippelhofer A, Hölling H. Befragung zur körperlichen Gesundheit im Rahmen des Kinder- und Jugendgesundheitssurveys. Gesundheitswesen 2002; 64 (Sonderheft 1): 17-22

${ }^{16}$ Prüfer P, Rexroth M. Verfahren zur Evaluation von Survey-Fragen: Ein Überblick. ZUMA-Nachrichten 1996; 39: 95-116

${ }^{17}$ Schenk L. Migrantenspezifische Teilnahmebarrieren und Zugangsmöglichkeiten im Kinder- und Jugendgesundheitssurvey. Gesundheitswesen 2002; 64 (Sonderheft 1): 59-68

${ }^{18}$ Schulz C, Becker K, Seiwert M. Kinder-Umwelt-Survey. Gesundheitswesen 2002; 64 (Sonderheft 1): 69-79

${ }^{19}$ Thefeld W, Bergmann KE, Burger M et al. Der Kinder- und Jugendgesundheitssurvey: Ermittlung des Gesundheitsverhaltens von Eltern und Kindern. Gesundheitswesen 2002; 64 (Sonderheft 1): 36-42 